\title{
The Activities of Bacterial Pathogens in Vivo
}

Based on contributions to a Royal Society Discussion Meeting 
This page is intentionally left blank 


\section{Editors}

\section{H. Smith}

Birmingham University, $U K$

\section{J. Dorman}

University of Dublin, $U K$

\section{G. Dougan}

Imperial College, UK

D. W. Holden

Imperial College, UK

\section{P. Williams}

University of Nottingham, $U K$ 


\section{Published by}

Imperial College Press

57 Shelton Street

Covent Garden

London WC2H 9HE

\section{Distributed by}

World Scientific Publishing Co. Pte. Ltd.

P O Box 128, Farrer Road, Singapore 912805

USA office: Suite 1B, 1060 Main Street, River Edge, NJ 07661

UK office: 57 Shelton Street, Covent Garden, London WC2H 9HE

\section{British Library Cataloguing-in-Publication Data}

A catalogue record for this book is available from the British Library.

\section{THE ACTIVITIES OF BACTERIAL PATHOGENS IN VIVO}

First published in the Philosophical Transactions of The Royal Society series B in 2000

First published by Imperial College Press in 2001

Copyright $(2000$ The Royal Society

The moral rights of the authors have been asserted.

All rights reserved.

ISBN $1-86094-272-5$

Printed in Singapore. 


\section{PREFACE}

Pathogenic bacteria, i.e. those that produce disease, have unique biological properties, which enable them to invade a host and produce sickness. The molecular bases of these biological properties are the determinants of pathogenicity and research objectives are to recognize them, identify them chemically and relate their structure to function. Most of our present knowledge comes from studies with cultures in vitro. However, there is a rising interest in bacterial behaviour in the infected host and new methods have been developed for studying it. The objective of the Discussion Meeting was to describe these methods and to show how they, and a recent surge in conventional studies, are shedding light on the activities of bacterial pathogens in vivo. Participants were asked to enquire about bacterial and host factors that operate in vivo to bring about sickness, to show how phenomena recognized in vitro relate to behaviour in vivo and, if evidence of relevance is not available now, to indicate how it might be obtained.

There are two introductory papers. The first, by Smith, outlines the new methods, poses questions about the behaviour of bacterial pathogens in vivo and indicates how answers may be obtained. Growth in vivo and the underpinning processes of nutrition and metabolism are given special emphasis because new methods are highlighting their importance. The second, Marshall et al., describes how the cellular environment can affect gene expression. It deals with the expression of genes coding for determinants of DNA topology (DNA gyrase, integration host factor and the nucleoidassociated protein H-NS) during adaptation of Salmonella typhimurium to the intracellular environment of macrophages. These global systems influence the transcription of genes involved in virulence, e.g. the $s p v$ locus. Next, five papers describe the new methods and their use in understanding hostpathogen interactions. The first, by Philpott et al., shows how a combination of studies with cell cultures and those with various animal models (infections of macaques, rabbit intestinal loops and murine lungs) have defined the molecular basis for mucosal invasion and the stimulation of inflammation by Shigella flexneri, which may apply to dysentry in man. In the second, Merrell \& Camilli describe the use of recombinase-based in vivo expression technology (IVET) to detect genes that are transcription- 
ally induced during infection, including those expressed transiently or at low levels. Spatial and temporal expression of specific genes, e.g. for the toxin co-regulated pilus $(t c p A)$ and cholera toxin $(c x t A)$ can be monitored during the course of infection. Hautefort \& Hinton discuss many techniques, other than IVET, for detecting gene expression in vivo, e.g. differential fluorescence induction and in vivo antigen technology (IVIAT). Some of these approaches can determine whether genes are expressed constitutively or in an organ-specific or cell-type-specific fashion. The paper by Unsworth \& Holden describes signature-tagged mutagenesis and its use for $S$. $t y$ phimurium in a mouse model to identify many virulence genes required for growth in vivo, including several clustered on a chromosomal pathogenicity island. It also shows how the use of a temperature-sensitive, non-replicating plasmid and competitive index tests can demonstrate that virulence gene function in vivo may differ from that predicted from in vitro studies. Finlay \& Brumell describe the interaction of $S$. typhimurium with relevant host cells both in vitro and in various animal models. Sophisticated imaging and molecular genetic tools are being used to monitor gene expression in both the pathogen and the host cell during infection. Tissue culture results have been confirmed and new questions evoked.

Three papers discuss the impact of the new methods. The first of these, by Heithoff et al., describes identification by IVET of many housekeeping and virulence genes of $S$. typhimurium, which are induced only in vivo. Some of these genes are expressed in vitro if regulatory genes of the DNA adenine methylase system (Dam) are mutated. Dam-negative mutants illustrate how the loss of a single enzyme can completely block the ability of a pathogen to cause disease yet fully elicit a protective immune response. The paper by Moxon \& Tang shows how a combination of genomics and methods for detecting gene expression in vivo are identifying genes that relate to virulence. It discusses practical and semantic difficulties in distinguishing between classical virulence factors and those that promote survival and growth in the host. It underlines the problem of obtaining animal models that reflect disease in the natural host. The paper by DiRita et al. relates knowledge of virulence gene regulation gained from studies in vitro to what occurs in vivo for two pathogens. For Vibrio cholerae, the ToxR regulon is active in vivo but the environmental factors that activate it are not clear. For Streptococcus pyogenes, capsule production occurs in an animal model of necrotizing skin infection. It is critical for virulence but dependent on 
mutation in a two-component regulatory system $\mathrm{CsrR}$ and $\mathrm{CsrS}$, i.e. on the loss of the regulation that occurs in vitro.

The next three papers discuss important aspects of bacterial pathogenicity and the evidence for their operation in vivo. Williams et al. describes quorum sensing, i.e. the regulation of bacterial processes in a celldensity-dependent manner through cell-to-cell communication by signalling molecules. Relevant signalling molecules have been detected in animal models and human infections. These molecules not only control bacterial gene expression but can also modulate host-cell responses. The paper by Cornelis describes the Yop virulon of Yersinia species as an archetype for type III secretion systems, which are activated by contact with eukaryotic cells. They allow bacteria to inject their proteins across two bacterial membranes and the host-cell membrane to destroy or subvert target cells. Studies with macrophages are described, but proof that they operate during animal infections has not yet been obtained. Morschhauser et al. shows that point mutation, genetic rearrangements and horizontal gene transfer processes contribute to macroevolution, long-term processes leading to new species, and microevolution, short-term developments occurring in days or weeks. Microevolution occurs in vivo; genome variability of pathogenic microbes leads to new phenotypes, which are important in acute development of an infectious disease. Horizontal transfer in vivo of genes by plasmids, bacteriophages and pathogenicity islands is more important for macroevolution.

The final paper, by Dougan et al., raises practical implications of the new knowledge. It deals with the handling of mucosally delivered antigens in attempts to design effective vaccines. Studies are needed of the mechanism of pathogenicity employed by microbial pathogens, of the combined mucosal and systemic immune response associated with infection and recovery, and of the mechanism of action of known good mucosal immunogens. The importance of studies in the natural host or whole animal systems is emphasized.

Some important aspects that emerged during the Meeting are summarized. Many of the new methods for studying bacterial behaviour in vivo require the pathogen to have robust genetics capable of easy manipulation. This is not always so, for example for Campylobacter jejuni, and it is fortunate that some methods, such as IVIAT, can be applied to such pathogens. The current surge in knowledge of bacterial behaviour in vivo comes as much from application of conventional methods (chemical and biological 
comparison of in vivo- and in vitro-grown organisms, mutation, virulence tests and complementation) as from new methods. A serious problem in applying both the new and conventional methods is the frequent lack of realistic animal models for human infections. This may severely limit our ability to get to the molecular basis of pathogenicity in humans. The problem may be mitigated in the future by the use of transgenic animals and the design of non-invasive methods for possible use in humans. Even when satisfactory animal models are available, better methods are needed for following the progress of infection spacially and in real time in situ. Because of its convenience, infection of macrophages in culture has been used as a halfway house between in vitro and in vivo conditions. Although these experiments may not reflect all the nuances of infection in animals, much useful information has been obtained from them, some of which has been confirmed by experiments with animals. At present, attention is largely concentrated on bacterial activities in vivo rather than the host factors that affect them and the interaction between the two. Most references to host factors are made in relation to the environment in macrophages or animals as a whole, rather than to specific factors and their changes during infection.

In the future, host DNA microarrays may be used to investigate global changes in eukaryotic gene expression in response to bacterial infection. Bacterial pathogens and their exoproducts are excellent probes for host cell biology. Some of the assumptions about the behaviour of pathogens in vivo based on research in vitro have been confirmed, particularly with regard to virulence determinants and regulatory systems. However, other assumptions have been shown to be too simplistic, e.g. operation of the ToxR regulatory system. Many of the genes expressed in vivo detected by the new methods are involved with nutrition, growth, metabolism and survival in the tissues of the host. Some well-known traditional virulence determinants - aggressins and toxins - have not been detected. Pathogens that are fully host adapted employ slip-strand mispairing to generate population diversity and have fewer transcription regulators than pathogens with both host and environmental lifestyles. We have moved from an era of the gene to the era of the genome and can now undertake 'top-down' approaches to problems of pathogenicity. Application of the new knowledge to the design of novel approaches to preventive therapeutic medicine has begun and will accelerate. Ways of inducing pathogen attenuation rather 
than death may be derived. Many of the genes detected by the new methods are not known to be involved in metabolism, stress response, regulation or virulence-determinant production by the pathogen under consideration, nor of any other bacterial pathogen. Thus, vast areas of the behaviour of pathogens in vivo remain unexplained. A major challenge for the future will be integration of the vast amount of information that will accumulate from genomics with equally voluminous data derived from intensive use of the new methodologies for studying bacterial behaviour in vivo.

March 2000
C. J. Dorman ${ }^{1}$
G. Dougan ${ }^{2}$
D. W. Holden ${ }^{2}$
P. Williams ${ }^{3}$
H. Smith ${ }^{4}$

${ }^{1}$ Department of Microbiology, Moyne Institute of Preventive Medicine, University of Dublin, Trinity College, Dublin 2, Republic of Ireland

${ }^{2}$ Department of Biochemistry, Wolfson Laboratories, Imperial College of Science, Technology and Medicine, London SW7 2AY, UK

${ }^{3}$ School of Pharmaceutical Sciences, University of Nottingham, University Park, Nottingham NG7 2RD, UK

${ }^{4}$ The Medical School, University of Birmingham, Birmingham B15 2TT, UK 
This page is intentionally left blank 


\section{CONTENTS}

Preface $\quad$ V

\section{Introduction}

Questions about the Behaviour of Bacterial Pathogens in Vivo

H. Smith

DNA Topology and Adaptation of Salmonella typhimurium to an Intracellular Environment

D. G. Marshall, C. J. Dorman, F. Bowe, C. Hale and G. Dougan

New Methods for Studying Bacterial Behaviour in Vivo

The Pathogenesis of Shigella flexneri Infection: Lessons from in Vitro and in Vivo Studies

D. J. Philpott, J. D. Edgeworth and P. J. Sansonetti

Detection and Analysis of Gene Expression During Infection by in Vivo Expression Technology

D. S. Merrell and A. Camilli

Measurement of Bacterial Gene Expression in Vivo

I. Hautefort and J. C. D. Hinton

Identification and Analysis of Bacterial Virulence Genes in Vivo

K. E. Unsworth and D. W. Holden

Salmonella Interactions with Host Cells: In Vitro to in Vivo

B. B. Finlay and J. H. Brumell 
In Yivo Gene Expression and the Adaptive Response: From Pathogenesis to Vaccines and Antimicrobials

D. M. Heithoff, R. L. Sinsheimer, D. A. Low and M. J. Mahan

Challenge of Investigating Biologically Relevant Functions of Virulence Factors in Bacterial Pathogens

R. Moxon and C. Tang

Virulence Gene Regulation Inside and Outside

V. J. DiRita, N. C. Engleberg, A. Heath, A. Miller,

J. A. Crawford and R. Yu

\section{Evidence for Operation in Vivo of Aspects of} Pathogenicity Revealed by Recent Work in Vitro: Potential Use of New Methods

Quorum Sensing and the Population-dependent Control of Virulence

P. Williams, M. Camara, A. Hardman, S. Swift, V. J. Hope,

K. Winzer, B. Middleton, D. I. Pritchard, B. W. Bycroft and D. Milton

Type III Secretion: A Bacterial Device for Close Combat with Cells of Their Eukaryotic Host

G. R. Cornelis

Evolution of Microbial Pathogens

J. Morschhäuser, G. Köhler, W. Ziebuhr, G. Blum-Oehler,

U. Dobrindt and J. Hacker

The Immune Responses to Bacterial Antigens Encountered in Vivo at Mucosal Surfaces

G. Dougan, M. Ghaem-Maghami, D. Pickard, G. Frankel,

G. Douce, S. Clare, S. Dunstan and C. Simmons 